\title{
Propuestas para la investigación cualitativa en educación artística
}

José Ignacio Bolaños-Motta

orcid.org/0000-0002-9976-1808

Universidad de los Llanos, Colombia

jbolanos@unillanos.edu.co
Mónica Alexandra Pérez-Rodríguez

orcid.org/0000-0001-8394-4848

Conservatorio de la Orinoquía

Escuela de Música Jorge Eliécer Gaitán, Colombia

\section{Resumen}

El presente artículo de reflexión dimensiona, en el ámbito de la educación artística, tres posibilidades metodológicas que se pueden desarrollar en contextos donde se adelantan programas de formación artística en cartografia social artística, investigación acción e investigación creación. El escrito explica, en una visión panorámica, algunas de las ventajas y complejidades posibles en cada una de estos tres enfoques metodológicos de investigación en el arte, para exponer finalmente la necesidad de pluralizar y ampliar la gama de posibilidades investigativas, ayudando a orientar de esta manera la construcción del conocimiento en el campo de la educación artística, a partir del ideal de la pluralización de las metodologías cualitativas, y dimensionando la posibilidad a futuro de que el arte construya su propio método de investigación.

\section{Palabras clave (fuente: tesauro de la UnEsco)}

Cartografia; creación artística; educación artística; investigación; metodología.

Recepción: 25/07/2018 | Envío a pares: 09/02/2019 | Aceptación por pares: 22/02/2019 | Aprobación: 24/04/2019 


\title{
Proposals for Qualitative Research in Arts Education
}

\begin{abstract}
This reflection paper evaluates three methodological options in the field of arts education that can be implemented in contexts in which artistic training programs in artistic social cartography, action research and creation research are offered. It provides an overview of the advantages and possible complexities of these methodological approaches to art research. Finally, it explains the need to multiply and expand the range of research opportunities, thus helping to guide knowledge creation in arts education, from the ideal of qualitative methodology pluralization, and exploring the future possibility that art builds its own research method.
\end{abstract}

Keywords (Source: UnEsco Thesaurus)

Cartography; artistic creation; artistic education; research; methodology. 


\title{
Propostas para a pesquisa qualitativa em educação artística
}

\begin{abstract}
Resumo
Este artigo de reflexão dimensiona, no âmbito da educação artística, três possibilidades metodológicas que podem ser desenvolvidas em contextos em que são realizados programas de formação artística em cartografia social artística, pesquisa-ação e pesquisa-criação. Este texto explica, em uma visão panorâmica, algumas das vantagens e complexidades possiveis em cada uma dessas três abordagens metodológicas de pesquisa em arte, para expor, finalmente, a necessidade de pluralizar e ampliar as possibilidades de pesquisa, ajudando a orientar, dessa maneira, a construção do conhecimento no campo da educação artística, a partir do ideal da pluralização das metodologias qualitativas, e dimensionando a possibilidade, no futuro, de que a arte construa seu próprio método de pesquisa.
\end{abstract}

Palavras-chave (Fonte: Tesauro da Unesco)

Cartografia, criação artística, educação artística, pesquisa, metodología. 
El método más perfecto será, pues, el que muestra [...] cómo debe dirigirse el espíritu. Asi se verá fácilmente cómo el espiritu, a medida que su conocimiento abarca más cosas, adquiere nuevos instrumentos que le permiten avanzar con mayor facilidad. (Spinoza, 1661, p. 19)

A partir de los aportes generados desde la década de 1980 en Estados Unidos por parte del Centro Getty para educadores en Artes Visuales de Los Ángeles (California), se logró desarrollar la denominada disciplina basada en educación en arte (Discipline-Based Art Education - DBAE) (Rodríguez, 2011). El progreso ininterrumpido de esta propuesta disciplinar hizo que en el continente europeo y en países como España y Brasil se desarrollaran nuevos estudios sobre el arte en la educación o, como se denomina en la actualidad, la Educación Artística. Hacia el año 2006, la Unesco expide una hoja de ruta para esta, como resultado de las propuestas realizadas en el marco de la Conferencia Mundial sobre la Educación Artística, celebrada con antelación en Lisboa, Portugal. Ese texto, de una u otra forma, ayudó a consolidar el planteamiento del para qué de la educación artística, lo cual la llevaría a convertirse en una de las necesidades educativas de los Estados nacionales demócratas de principios del siglo XXI (Unesco, 2006). Lo anterior parte de la premisa de que el arte ayuda al ser humano a desarrollar la mente hacia labores complejas y necesarias para la sociedad de la información (Castells, 1996), propuesta para el siglo XXI. Ante la creciente necesidad del fomento de la educación artística en las diversas latitudes, se empezaron a desarrollar programas de investigación encaminados a fortalecer el ejercicio de la investigación en educación artística. Fue de esta manera como el arte, que a lo largo de toda la historia había sido un mero acto de construcción de la realidad (Abad, 2011), se legitimó como uno de los componentes necesarios en los programas educativos, ello como una propuesta orientada a erradicar el analfabetismo estético (Aguirre, Jiménez y Pimentel, 2011) a partir de un modelo educativo desarrollado desde la escuela.
Así las cosas, desde no hace muchos años se han venido desarrollando profesionales del arte, de quienes no solo se espera que sientan el arte, lo interpreten o lo realicen, sino también que sean artistas-maestros, que a partir de un ejercicio investigativo piensen el arte o, dicho en otras palabras, maestros capaces de pensar el arte para poderlo investigar. De manera paralela a lo anteriormente expuesto, durante los últimos años la educación artística ha venido transformándose y creciendo, y ha empezado un proceso de autonomía gracias a las investigaciones que han dado lugar a una profundización del arte en la educación (Aguirre et al., 2011), lo cual ha impactado de manera paralela a múltiples países de Europa y de América del Sur, en donde se han generado también diversos avances que han justificado teóricamente el arte en el mundo de la educación y la pedagogía. Para entrar un poco en el problema de la disciplina como tal, el presente artículo enfatiza tres metodologías de investigación dentro del campo de la educación artística.

Dichas metodologías buscan ampliar las posibilidades investigativas, dado que el artista se encuentra ante una doble necesidad: la de ser artista y la de investigar el arte, y esta dicotomía puede dar lugar al surgimiento de múltiples problemas dentro de la investigación, en lo que respecta a los procesos investigativos en educación artística. Lo anterior, incluso a partir de la misma formulación del problema a investigar, visto que "más del 50\% de los estudiantes desconoce las metodologías de investigación en educación artística; el 50\% no define ni delimita problema de investigación; el 30\% presenta dificultades en la formulación y limitación de objetivos; el 58\% no realiza un estado del arte sobre el tema antes de ejecutar sus proyectos" (Uribe, 2012, p. 24).

Ante lo expuesto, este artículo buscará, en planos muy generales, presentar algunos caminos posibles dentro del enfoque de la investigación cualitativa (Sandin, 2003) y en el ámbito de la educación artística. En consecuencia, se proponen tres enfoques heredados de las ciencias sociales: la car- 
tografía social, la investigación acción y, finalmente, como un método propio de la investigación en educación artística, la investigación creación, de la cual se presentarán algunas descripciones y propuestas aún por construir.

\section{La cartografía social artística}

La cartografía puramente dicha se configuró desde sus inicios como una estrategia de recolección de información que pretendió mostrar una imagen factual de la realidad terrestre, a través de los planos que el ser humano desarrollaba en la disciplina de la geografía (Stromquist, 2006) y con el fin de evidenciar los límites de los Estados nacionales. En consecuencia, los planos desarrollados a partir de los ejercicios cartográficos, de una u otra forma, se convirtieron en agentes claves para los poderes establecidos en las distintas periferias del mundo. Para las ciencias sociales se ha empezado a desarrollar en los últimos años un nuevo enfoque denominado cartografía social. Este ejercicio comenzó como una estrategia de recolección de la información destinada a graficar la construcción de las problemáticas o significaciones de vida y territorio del ser humano, y esta técnica suele usarse en el problema de investigación dentro de determinado núcleo poblacional. Dada la fuerza que ha tomado la cartografía social en Latinoamérica, esta forma de investigación ha pasado de ser una estrategia de recolección de la información a convertirse en una metodología de investigación (Vélez, Rátiva y Varela, 2012). La popularidad de este ejercicio investigativo puede deberse a que posibilita la construcción de mapas de pensamiento, en un enfoque que, además de didáctico, resulta ser "colectivo, horizontal y participativo" (Diez, Escudero y carballeda, 2012, p. 14).

La cartografía social propiamente dicha se ha venido desarrollando en distintas disciplinas $y$, de manera muy especial, en el mundo de la educación y la pedagogía, a tal punto que ha pasado a vincularse de manera directa con los desarrollos de la investigación en educación, denominándose enton- ces como cartografía social pedagógica (CSP), de la cual se señala que, en toda "ruta emergente de investigación cualitativa, [ella] puede utilizarse como opción metodológica de investigación en educación y pedagogía (...) [ya que] abre horizontes didácticos que permiten resignificar las prácticas de diversos actores educativos [...] configurar lazos de pertenencia a una colectividad o a formas de comprender la educación" (Barragán, 2016, p. 280)

Y es así como, de manera casi simultánea a la cartografía social pedagógica, en la que se intenta trasladar ciertos aspectos de la cartografía social al campo educativo, en cuanto vía para transformar la educación (Barragán, 2016), ha nacido la cartografía social artística, la cual pretende abrirse camino en el ámbito de los procesos de indagación en el arte. Sobre esta se puede decir que pretende desarrollar análisis sobre el simbolismo pictórico o discursivo, lo cual da lugar a que este simbolismo se convierta en una posibilidad interpretativa y traducible. En consecuencia, la cartografía artística "es activa en cuanto va abriendo y cerrando relaciones en la medida en que va conteniendo y liberando las fuerzas y sus sensaciones [...] crea un cuerpo de sensación, un cuerpo que, hablando estrictamente, no es ni un objeto (obra de arte) ni un sujeto (su experiencia), sino una actualización de fuerza que acompasa obra y espectador, emergiendo de acuerdo con sus condiciones locales" (Zepke, 2009, p. 295).

En concordancia con lo anterior, en una primera instancia se relativizaría el concepto que se tiene de expresión plástica o artística, pues pasaría a convertirse en un dato, según lo establece la cartografía social; dato desarrollado en segunda instancia como un cuerpo sensible o una experiencia estética (Tatarkiewicz, 2001), que puede ser elevado y convertido a una expresión artística. Dicha cartografía habrá de ir acompañada de una pregunta dirigida al interior del artista investigador, pregunta que puede estar plasmada sobre el papel en el cual se va a hacer la cartografía, o también ser expresada de forma oral por quien preside el acto investigativo. 
Continuando con lo expuesto, y ya en la disciplina artística de la música, resulta ideal que esta pregunta esté formulada como una auto-pregunta del tipo: ¿qué imágenes vienen a mi mente cuando escucho la música judía? (Bolaños, 2016). Si esta pregunta fuera elaborada para un trabajo con una comunidad religiosa judía, con base en lo expuesto aquí ella puede ser desarrollada dentro de cualquier disciplina en la que se quieran levantar datos', sea en los espacios de la enseñanza de la plástica, de la música o de las artes escénicas. En otras palabras, dentro de una jornada de educación artística vista de forma muy general.

Aunque es de aclarar que este ejercicio investigativo se relaciona de manera directa con la expresión plástica, como se había dicho, el método se ha desarrollado por fuera de las artes (Diez et al., 2012), y dada la similitud entre la práctica en las artes y fuera de ellas, es claro que el éxito de la cartografía depende de las preguntas que orientan su trabajo (Barragán, 2016). Dicho esto en otras palabras, el dato investigativo y la expresión artística pasarían a ser ontológicamente una misma cosa, sin importar la intensión inicial. En este plano de ideas, el pedagogo estadounidense de origen judío Howard Gardner (1997) explica que una expresión pictórica arroja un simbolismo discursivo que opera en el orden de la sensación y de lo significable. Y es precisamente sobre este simbolismo discursivo donde entran a operar las pupilas del investigador decodificando los datos presentes en la expresión artística del actor de investigación.

Para ampliar la complejidad de lo expuesto, Sautu, Boniolo y Dalle (2005, p. 14) explican que "los datos solo logran hablar cuando la teoría les aporta el don del lenguaje", hecho por el cual la cartografía social artística han de desarrollarla investigadores con capacidad para trabajar con saberes emergentes y a partir de las obras de arte o, como se había dicho,

$1 \quad$ Un ejemplo similar fue aplicado en otra investigación en donde la pregunta a la cartografía social artística fue: ¿qué imágenes vienen a mi mente cuando escucho las palabras cultura llanera? (Bolaños, 2018). teorías que posibiliten la explicación de los datos hallados en la cartografía, asumida como una expresión artística. Ante esto, la cartografía social artística daría entonces la posibilidad de convertirse en un modelo de interpretación de la realidad sensible y subjetiva de quien se dice ser artista e investigador. La posibilidad aquí planteada se ha de zanjar a partir de trabajos de grado, de pregrado y de posgrado, prácticas investigativas y demás tipos de investigaciones académicas, con el objetivo de generar un impacto en los programas de formación de los educadores artísticos del país. Asumir la cartografía artística como una metodología de investigación es una posibilidad de gran magnitud para la educación artística, pues a través de ella se hace posible visibilizar de manera directa las dimensiones simbólicas que habitan en la memoria o en las vivencias (Dilthey, 2015) del ser del sujeto. En otras palabras, es una posibilidad más para la expresión del ser de quien desarrolla este ejercicio. Por tal razón, en el ámbito de las artes la cartografía permite "comprender la memoria desde su dimensión cartográfica [...] contribuyendo en la creación de una nueva poética sobre la realidad que se teje a través de los discursos, las acciones, los objetos y, en general, a través de diferentes procesos semiósicoartísticos" (Agudelo, 2015, p. 148).

Lo perceptible dentro de esta metodología es el hecho de integrar la técnica del dibujo (Agudelo, 2015) con la reflexión conceptual sobre la memoria personal y experiencial; en consecuencia, se puede construir el contexto a través del símbolo y de la interacción desarrollada con el grupo poblacional encargado de la cartografía; asimismo, se puede develar cada uno de los trazos que dieron origen al dato desde la subjetividad. La cartografía artística se configura entonces como un mapa que ayuda a estructurar la subjetividad pura de los individuos, en tanto no solo cobre expresión en la figura, sino también en el tratamiento colectivo que se le da a la misma², a través del ejercicio artístico. Frente a la

2 Aunque no se descarta realizar este mismo ejercicio de manera individual, la importancia radica más en la expresión colectiva de las comunidades. 
metodología expuesta se recalca que no es poco el recorrido histórico del concepto de mapa desde la visión de Ptolomeo hasta el uso popularizado por Google Maps. Dichas transformaciones tecnológicas han dado al ser humano la posibilidad de repensar el uso del mapa y han llevado a que la cartografía social artística transforme el concepto de mapa como lugar importante para la subjetividad, pues, al involucrar aspectos como el color, la perspectiva y la intensión, sugiere una nueva cosmovisión del espacio que parte del individuo hacia el universo que lo rodea y no exactamente al contrario. Dicho esto en otras palabras, los nuevos cartógrafos ya no se dirigen a los mapas con el objetivo de indicarle al ser humano dónde se encuentran, puesto que, en una visión más subjetiva, los mapas señalan que ellos se forman "alrededor de nosotros, y sobre la marcha" (Perales, 2010, p. 88).

La cartografía social es un aporte clave para los procesos investigativos en el arte, en tanto que a través de ella la expresión del ser se ve objetivada como dato o conjunto de datos a decodificar a través del diálogo investigador-comunidad. Sobre lo anterior es de recalcar que la valía de esta metodología se halla en que es precisamente el artista el encargado de la investigación, y en particular se podría decir que el artista plástico estaría en competencia para interpretar el gráfico desarrollado por la comunidad o de quien se dice encargado de la construcción del símbolo o expresión inscrita en la cartografía. Ello porque, en concordancia con Kandinsky, "toda forma tiene pues un contenido interno del cual es expresión" (1989, p. 49) y el artista vendría a encargarse de la correspondiente interpretación. La cartografía social artística, desarrollada como metodología o aun como estrategia de recolección de la información, resulta ser de gran valía para la investigación en educación artística, y aunque en determinado momento se podría pensar que este método se encuentra más ubicado en el campo de la plástica que en el campo de la música o en las artes de la corporeidad (teatro y la danza), este ejercicio investigativo y hermenéutico puede ser realizado en los procesos de enseñanza de cualquiera de las vertientes del arte en general. La cartografía ayuda a dar forma a la reflexión sobre la vivencia (Dilthey, 2015) realizada por el artista, pues en este ejercicio se hacen presentes dos expresiones: la expresión artística y la expresión de la cotidianidad cultural del ser (Aguirre et al., 2011).

\section{La investigación acción (IA) en educación artística}

La definición de investigación acción (Acción Research) fue enunciada por primera vez por el psicólogo Kurt Lewin (1946) a mediados del siglo anterior. Esta propuesta tenía por objeto generar una práctica reflexiva social enfocada a transformar las prácticas sociales, idea que ha ido evolucionando en la actualidad y se podría definir como el "estudio de una situación social con el fin de mejorar la calidad de la acción dentro de la misma" (Elliott, citado por Rodríguez et al., 2011, p. 4). Sobre esto, se agrega: "los principales beneficios de la investigación acción son la mejora de la práctica, la comprensión de la práctica y la mejora de la situación en la que tiene lugar la práctica. La investigación-acción se propone mejorar la educación a través del cambio y aprender a partir de las consecuencias de los cambios" (Latorre, 2005, p. 27).

La propuesta denominada Acción Research resultó de tan amplio uso que generó toda una tradición investigativa (Sandín, 2003) en torno a este modelo de trabajo con comunidades. Este modelo de trabajo trascendió lo sociológico para dar lugar a estudios en otras disciplinas de las ciencias sociales, y también de manera muy consistente en el campo de la educación, en donde a través de este método se hizo posible un conocimiento profesional, a partir de la reflexión sobre la práctica educativa (Elliott, 2000). Las figuras más relevantes que continuaron con esta tradición en la metodología de la investigación son, entre otras: Carr y Kemmis (1986), Kolb (1984), Elliott (2000) y Latorre (2005). 
A partir de este enfoque se han generado nuevos intereses y bifurcaciones, como es el caso de la investigación acción educativa (IAE) que se propone como una línea de investigación en torno al saber pedagógico (Restrepo, 2004) y la investigación acción participativa (IAP) (Fals, 2014), enfocada de manera más profunda a trabajar con comunidades. Partiendo del presupuesto de que la investigaciónacción ha estado enfocada a la transformación de las prácticas de vida, se asume como primera propuesta que es posible transformar las prácticas cotidianas de la escuela tradicional a partir de una práctica artística, bien sea musical o corpórea sensitiva (como la danza o el teatro), o a partir de la expresión plástica, desarrollada como una forma de lenguaje visual (Acaso, 2009). De tal suerte, la investigaciónacción tendría lugar dentro de la investigación en la escuela tradicional, dado que a través de la IA es posible desarrollar prácticas artísticas encaminadas a transformar la realidad escolar a través del arte en la educación. De manera paralela con lo expuesto se plantea una segunda propuesta, en tanto que la IA hace posible desarrollar múltiples estudios sobre la base de la práctica artística, con el objetivo de mejorarla, lo cual da lugar a una investigaciónacción para el arte, como un fin en sí mimo. A partir de los aportes de Elliott (2000) en el ámbito de la investigación-acción sobre las escuelas, se podría fundamentar un trabajo similar, pero en el ámbito de la investigación en educación artística, y, en concordancia con lo expuesto por Elliott, en tres etapas: la primera etapa consistiría en la implementación de una propuesta investigativa centrada en determinado problema del contexto artístico o dirigida a la promoción de los valores artísticos educativos en el aula. Luego, en una segunda etapa, resultaría válida la formulación de un grupo de hipótesis cualitativas desarrolladas dentro de la práctica artística implementada por grupos sociales ligados al arte. Finalmente, se daría una tercera etapa, cuyo eje es la actividad artística y su correspondiente evaluación de las estrategias de acción.
En términos generales, el aporte de la IA a la enseñanza de las artes radica en la posición que asume este modelo de investigación frente a las actividades del ser humano dentro de un núcleo social determinado. En consecuencia, la IA, aplicada en el mundo del arte, consideraría las prácticas sociales artísticas como "actos de investigación". Ejemplos sobran en los grupos de teatro, danza y música, entre otros. Por lo expuesto, el modelo de la IA resulta ser de gran valía para la educación artística, quizás con mucha más propiedad que para las artes propiamente dichas, dado su enfoque directamente relacionado con el hecho educativo, pues:

El conocimiento-en-acción práctico del profesional es de carácter ético más que técnico, o sea, un conocimiento de cómo realizar una forma ética [...] el conocimiento-en-acción profesional [...] ayuda al profesional a plantear de antemano las posibilidades futuras, pero no a predecirlas, y siempre ha de mantenerse abierto a la sorpresa, a la necesidad de plantearse y reflexionar sobre la adecuación de su conocimiento a la situación presente. (Elliott, 2000, p. 14)

Queda entonces en manos del artista e investigador cualitativo el desarrollo de las fases de la investigación artística, en cualquiera de las dos formas o acepciones expuestas anteriormente. Sobre lo anterior también es válido decir que, dada la fuerza y tradición del modelo IA, enfoques metodológicos como los descritos en este artículo pueden ser de gran utilidad cuando los artistas realicen investigación o produzcan conocimiento científico desde su saber, para una posterior productividad en revistas relacionadas con temáticas como la didáctica, la lúdica, la recreación, el tiempo libre y la educación en general. Las posibilidades a indagar son muy amplias, pues a través de estudios desde la IA o la IAP se podrían adelantar investigaciones en torno al desarrollo del arte popular. Asimismo, por medio de la IA es posible transformar las prácticas educativas artísticas, como también las prácticas artísticas en sí mismas, y si bien los colectivos humanos artísticos 
poseen problemáticas, al igual que cualquier otro grupo humano, sobre estas prácticas de vida artística es posible dar lugar a nuevas reflexiones en torno a las alteridades y sonoridades (Muñoz, 2016) de los sujetos, todo ello a partir de los estudios realizados por artistas investigadores.

\section{Investigación creación}

Desde hace ya varios años se ha venido desarrollando la idea de la investigación-creación como una posibilidad investigativa desarrollada desde las artes y al servicio investigativo de las artes. A través de este enfoque, el investigador-artista pretende generar un conocimiento desde su propia disciplina (Daza, 2009). Con ello se valida la percepción artística como una forma de conocimiento académico y se vindica de esta manera el lugar del artista como ente promotor de saber (práctico o no). Así la percepción artística se valida como un producto de un saber o proceso investigativo, dado que en los últimos años ha tomado fuerza la idea de que el arte desarrolla el pensamiento de los pueblos. Sobre lo anterior, el ejercicio investigativo, en términos de su ejecución, permite que se lo vea como una actividad muy elemental, con un método de trabajo claro que "implica recogida de datos, recensión de la literatura que existe sobre el tema, definición de una problemática, recurso a una metodología y a conceptos definidos, utilización de un protocolo de análisis que permita la interpretación de datos [...]. Incluye necesariamente una parte de creación y una reflexión escrita que acompaña el trabajo de creación" (Féral, 2009, pp. 324-325).

En consecuencia, la producción de conocimiento a través del arte incluiría -entre otras formas de comunicación- la producción de documentos escritos3, pues "el concepto de producción implica, además, que los científicos toman ciertos 'insumos' o

3 Aquí valen en el campo de la música: las partituras. Solo que, para indagar la calidad de determinada creación, se necesita de curadurías, jurados o arbitrajes del arte, lo cual requiere una discusión más amplia. ingredientes y los transforman en 'productos' [...] el principal producto de la ciencia es el desarrollo de conocimientos sistemáticos y organizados, plasmados primero en escritos y otros documentos donde se comunican los resultados" (Maletta, 2009, p. 117).

El ejercicio de la investigación-creación le da pie al artista para que, en paralelo con la comunicación ya establecida entre él y su espectador, a través de la obra de arte escriba aquella experiencia por medio de la cual construyó su obra de arte, lo cual potencia su expresión y su obra. Así las cosas, existirían dos canales de comunicación: su obra escrita y su obra pura, lo cual, dicho en forma metafórica, equivale a decir que un ingeniero presenta en un primer momento la casa y, posteriormente, los planos de la casa. También se ha dicho que la educación artística presta un servicio insuficiente como vehículo de validación de la producción artística, puesto que aquello que se acepta como conocimiento no es precisamente el contenido estético de la obra, sino el escrito sobre ella realizado. La crítica radica en que toda creación artística debería ser vista como un producto de investigación (Osuna, 2012). Sobre lo anterior, y para desarrollar un poco esta idea, Hernández agrega:

El niño investiga cuando juega; el cocinero tiene un taller de investigación para preparar sus nuevas recetas; el actor investiga la construcción de su personaje; el artista investiga en su taller y lo refleja, [...] el cientifico investiga en su laboratorio o en el campo [...]. Lo cual significa que la expresión "investigación" forma parte del lenguaje cotidiano al igual que, en los últimos años, ha venido a serlo la noción de creatividad. (2006, pp. 19-20)

Sobre la investigación-creación hay un punto a tener en cuenta y es que crear no es tarea fácil, pues no todos los artistas hacen tal cosa y, de hecho, algunos solo recrean obras, como es el caso de la gran mayoría de músicos, quienes interpretan obras de compositores precedentes, en tanto que no es de su 
competencia componer nuevas obras, sino interpretarlas (o recrearlas). De manera similar, muchas personas del mundo del teatro desarrollan libretos escritos por literatos precedentes, sin verse en la necesidad de inventar nuevos libretos o guiones para determinada dramaturgia.

Dada la preocupación de algunas universidades del centro del país por la forma como sus artistas generan o tienen la posibilidad de generar conocimiento (Santamaría et al., 2011), y dadas las limitadas posibilidades institucionales del artista en cuanto a la producción académica se refiere, también se hace necesario desarrollar nuevas posibilidades para la educación artística a partir del campo de las metodologías de investigación cualitativa. Ante la mencionada necesidad, la valía de la IC radica en el hecho que este método es el más cercano a las artes, por cuanto proviene de ellas, hecho que lo particulariza de cara a los demás enfoques, que sí son heredados de la tradición de las metodologías (Sandín, 2003) con trayectoria previa en campo de las ciencias sociales y de la educación.

\section{A manera de conclusión}

Como se anunció, aquí se presentan tres métodos de investigación, de los cuales se resaltan los aportes metodológicos que cada uno de ellos ofrece para la correspondiente realización de investigaciones cualitativas en el campo de la educación artística. Por demás, se debe estimar que, tanto en la ciencia como en el arte, la creatividad es de vital importancia para el incremento de la producción académica en el campo del arte, a raíz de lo cual, las tres metodologías expuestas pueden pluralizar y ampliar la gama de posibilidades investigativas, favoreciéndose en consecuencia la construcción del conocimiento en el campo de la educación artística. Se asume entonces que las metodologías expuestas a lo largo del presente texto dan lugar a que muchos artistas no solo fortalezcan su producción artística sino que también hagan valiosos aportes a la escuela, al campo de la ciencia y al mundo de la educación en general.
No está de más decir que desde hace algunos años el arte, en sus diversas manifestaciones, ha venido ganando espacio en el campo de la investigación y afortunadamente ha logrado vindicarse como una de las formas de conocimiento. El argumento que indica que el arte es una forma de conocimiento que ayuda al desarrollo de los pueblos ha ganado adeptos en el mundo académico. Ello se evidencia también en las decisiones políticas sobre el mismo4. Por consiguiente, es ahora deber de los artistas y/o artistas e investigadores repensar el "ser" y el "método" de su saber hacer artístico.

La investigación acción y la cartografía social artística avanzan vertiginosamente en el campo de las ciencias humanas y en las ciencias de la educación, pero es posible que no se esté avanzando al mismo ritmo en el campo del arte, cuando, juntas constituyen, por sí mismas, una gran posibilidad. De otro lado, si bien la investigación creación ha tomado mucha fuerza en el arte, ante su diversidad de posibilidades, todas plausibles, se asumió en el presente texto aquella que se consideró más pertinente y con mejores posibilidades para los artistas-maestros en el campo de la educación.

En concordancia con lo anterior, es también necesaria una diversificación de metodologías cualitativas a desarrollar dentro de las propuestas investigativas destinadas al campo de educación artística. Esta labor les compete a los pregrados y maestrías que orientan este campo de saber, de tal forma que en el futuro se establezcan transversalidades o mixturas de métodos de investigación en el campo del arte. De tal suerte, la educación artística se encontraría ante la posibilidad de pluralizar los métodos de investigación para avanzar en ellos, hasta encontrar un método propio..., aquel que, guiado por lo ex-

4 Prueba de ello es que, hacia el mes de diciembre de 2018, el Departamento Administrativo de Ciencia, Tecnología e Innovación de Colombia (Colciencias) tomó la decisión de destinar espacios dentro de la denominada producción científica a los productos artísticos, en sus diversas formas: eventos, artefactos, obras, prácticas y demás actividades de nuevo conocimiento relacionado con el arte. 
puesto por Spinoza y parafraseando el encabezado de este escrito: sea el más perfecto para "dirigir el espíritu", adquiriendo "nuevos instrumentos que le permitan avanzar con mayor facilidad" sobre el campo del arte y la ciencia, lo cual hace un tiempo parecía impensable.

\section{Referencias}

Acaso, M. (2009). El lenguaje visual. Barcelona: Paidós.

Agudelo, P. (2015). Cartografías artísticas: reflexiones acerca de los juguetes y la memoria. Revista Virtual. Universidad Católica del Norte, 45, 143-158. Recuperado de http://revistavirtual.ucn.edu.co/index.php/RevistaUCN/article/view/663

Abad, J. (2011). Usos y funciones de las artes en la educación y el desarrollo humano. Madrid: Metas Educativas.

Aguirre, I., Jiménez, L. y Pimentel, L. (2011). Educación artística, cultura y ciudadanía. Madrid. Metas Educativas.

Barragán, D. (2016). Cartografía Social Pedagógica: Entre teoría y metodología. Revista Colombiana de Educación, 70, 247-285. Recuperado de http://www.scielo.org.co/scielo.php?pid=S0120-39162016000100012

Bolaños, J. (2016). El judaísmo sefardí en las comunidades judías Mesilat Yesharim y Or Va Derej,y sus prácticas musicales. Tesis de Maestría en Educación de la Universidad del Cauca, Popayán: Maestría en Educación Multicultural y Etnoeducación.

Bolaños, J. (2018). Procesos de adaptabilidad e identidad, al interior de las prácticas musicales en VillavicencioMeta. Entramado, 14(2), 182-196. DOI: 10.18041/1900-3803/entramado.2.4750

Carr, W.y Kemmis, S. (1986). Teoría crítica de la enseñanza. La investigación-acción en la formación del profesorado. Barcelona. Martínez Roca.

Castells, M. (1996). La era de la información: la sociedad red (vol. 1). Madrid: Alianza.

Daza, S. (2009) Investigación-creación. Un acercamiento a la investigación en las artes. Manizales. Plumilla Educativa.

Diez, J., Escudero, H. y Carballeda, A. (2012). Cartografía social. Investigación e intervención desde las ciencias sociales, métodos y experiencias de aplicación. Patagonia: Universitaria de la Patagonia.

Dilthey, W. (2015). Introducción a las ciencias del espíritu. México. Fondo de Cultura Económica.

Elliott, J. (2000). La investigación-acción en educación. Madrid: Morata.

Fals, O. (2014). Ciencia, compromiso y cambio social. Buenos Aires: El Colectivo.

Féral, J. (2009). Dosier “Scanner”. Recerca i creació. Estudis escènics: quaderns de l'Institut del Teatre. 
ISSN 0123-1294 | e-ISSN 2027-5358 | Educ.Educ. Vol. 22. No.1 | Enero-abril de 2019 | pp. 51-63.

Universidad de La Sabana | Facultad de Educación

Gardner, H. (1997). Arte, mente y cerebro. Una aproximación cognitiva a la creatividad. Buenos Aires: Paidós.

Hernández, F. (2006). Campos, temas y metodologías para la investigación relacionadas con las artes. Madrid: Omagraf.

Kandinsky (1989). De lo espiritual en el arte. México: Premia.

Kolb, D. (1984). Experiential learning: experience as the source of learning and development. Nueva Jersey: Prentice Hall.

Latorre, A. (2005). La investigación-acción. Conocer y cambiar la práctica educativa. Madrid, Imprimeix.

Lewin, K. (1946). La investigación-acción y los problemas de las minorías. Madrid: Editorial Popular.

Maletta, H. (2009). Epistemología aplicada: Metodología y técnica de la producción científica. Lima: Cepes - Universidad del Pacífico.

Muñoz, P. (2016). Las almas de los violines 'negros". Tesis Doctoral, Doctorado en Antropología de la Universidad del Cauca, Popayán.

Osuna, J. (2012). Un viaje a ninguna parte: la investigación-creación como vehículo de validación institucional de la producción artística. Cuadernos de Música, Artes Visuales y Artes Escénicas, 7(1), 5-9. Recuperado de https://revistas.javeriana.edu.co/index.php/cma/article/view/2351

Perales, V. (2010). Cartografías desde la perspectiva artística. Diseñar, trazar y navegar la contemporaneidad. Arte, Individuo y Sociedad, 22(2), 83-90. Recuperado de https://revistas.ucm.es/index.php/ARIS/article/ view/ARIS1010220083A

Read, H. (1982). Educación por el arte. Barcelona. Paidós.

Restrepo, B. (2004). La investigación-acción educativa y la construcción de saber pedagógico. Educación y Educadores, 7, 45-55. Recuperado de http://educacionyeducadores.unisabana.edu.co/index.php/eye/article/ view/548

Rodríguez, F. (2011). Construcción ciudadanía y educación artística. Madrid: Metas Educativas.

Rodríguez et al. (2011). Investigación acción. Métodos de investigación en educación especial. Libro digital.

Sandín, M. (2003). Investigación cualitativa en educación: fundamentos y tradiciones. Madrid: McGraw-Hill Interamericana.

Santamaría, C. et al. (2011). La productividad de las artes en las universidades colombianas: desafíos a los mecanismos de medición del conocimiento. Cuadernos de Música, Artes Visuales y Artes Escénicas, 6(2), 87-116. Recuperado de https://revistas.javeriana.edu.co/index.php/cma/article/view/1785

Sautu, R., Boniolo, P., Elbert, R.y Dalle, P. (2005). Manual de metodología. Buenos Aires: Clacso. 
Spinoza, B. (1661). Tratado de la Reforma del Entendimiento. www.philosophia.cl. Escuela de Filosofía Universidad Arcis.

Stromquist, N. (2006). Una cartografía social del género en educación. Educação \& Sociedade, 27(95), 361-383. DOI: 10.1590/S0101-73302006000200003

Tatarkiewicz, W (2001). Historia de seis ideas. Arte, belleza, forma, creatividad, mímesis, experiencia estética. Madrid. Tecnos.

Unesco (2006). Hoja de ruta para la educación artística. Conferencia Mundial sobre la Educación Artística: construir capacidades creativas para el siglo XXI. Lisboa: Unesco.

Uribe, J. (2012). La investigación en educación artística. Una guía para la presentación de proyectos de pregradoy postgrado. Bogotá: Universidad Distrital Francisco José de Caldas.

Vélez, I., Rátiva, S. y Varela, D. (2012). Cartografía social como metodología participativa y colaborativa de investigación en el territorio afrodescendiente de la cuenca alta del río Cauca. Revista Colombiana de Geografía, 21(2), 59-73. DOI: 10.15446/rcdg.v21n2.25774

Zepke, S. (2009). La cartografía artística de la sensación: tres obras recientes de Rosario López. Antípoda, 7, 295305. DOI: 10.7440/antipoda7.2008.12 